\title{
American College of Medical Genetics and Genomics: revised scope of practice and strategic plan
}

\author{
Gerald Feldman, MD, PhD, FACMG ${ }^{1-3}$
}

In this month's issue of Genetics in Medicine, you will find the revised Scope of Practice for medical geneticists, which includes clinical, laboratory, and $\mathrm{PhD}$ geneticists. This document was recently approved by the American College of Medical Genetics and Genomics (ACMG) Board of Directors and reflects the efforts of both the Board of Directors and members of ACMG, who had the opportunity to comment on the posted draft. Each comment was reviewed, and the document was revised accordingly. The present document replaces the original Scope of Practice, which was approved by the ACMG Board of Directors in 2008. Defining the practice of medical genetics has been and will continue to be a major activity of the ACMG because the organization is responsible to its members and to the public for defining the scope and standards of practice for our growing and rapidly changing specialty. The Board of Directors felt that it was critical to revise the original document to answer "who," "what," "where," "when," "why," and "how" in the current era of genomic medicine, which involves other critical genetics health professionals, such as genetic counselors, genetic nurses, and other laboratory professionals performing genetic and genomic testing. This Scope of Practice clearly delineates the value of a genetics consultation by a board-certified clinical geneticist and the importance of a board-certified clinical laboratory geneticist in laboratory genetic and genomic medicine, including their role in the interpretation of biochemical, cytogenetic, and molecular test results.

Our specialty is faced with many challenges, including legislative and regulatory debates on how best to incorporate the practice of genetic and genomic medicine and testing into everyday practice. We hope that payers, legislators, and rule makers responsible for these decisions will find this document relevant in guiding their decisions. We also hope that the document illustrates to the public and our patients that our profession strives to maintain the highest standards of medical and laboratory practice, that our specialty is uniquely situated to provide care in the emerging realm of genomic medicine, and that we must always act in the best interests of the patient. ACMG also recognizes that it is inevitable that other specialties may share some common ground with ours. This document will continue to evolve in the technology-driven field in which we practice.

Finally, I call your attention to the new ACMG Strategic Plan that is now posted on the ACMG website (http://www. acmg.net). The mission of ACMG has been refined as follows: "Developing and sustaining genetic and genomic initiatives in clinical and laboratory practice, education, and advocacy." There are three guiding pillars of the new plan: (i) clinical and laboratory practice-establish the paradigm of genomic medicine by issuing policy statements and evidence-based or expert clinical and laboratory practice guidelines and through descriptions of best practices for the delivery of genomic medicine; (ii) education-provide education and tools for medical geneticists, other health professionals, and the public and grow the genetics workforce; and (iii) advocacy-work with policy makers and payers to support the responsible application of genomics in medical practice.

These are indeed exciting times for ACMG and its members. We hope you find these documents helpful in defining our specialty and delineating the opportunities to follow.

\section{DISCLOSURE}

The author declares no conflict of interest.

\footnotetext{
${ }^{1}$ American College of Medical Genetics and Genomics, Bethesda, Maryland, USA; ${ }^{2}$ Department of Pediatrics, Center for Molecular Medicine and Genetics, Wayne State University School of Medicine, Detroit, Michigan, USA; ${ }^{3}$ Department of Pathology, Center for Molecular Medicine and Genetics, Wayne State University School of Medicine, Detroit, Michigan, USA. Correspondence: Jerry Feldman (gfeldman@med.wayne.edu) 